\title{
Casa hogar-asilo y su influencia en la calidad de vida del adulto mayor: revisión bibliográfica
}

\author{
Tizoc-Márquez, Arodi; Esquivel-Rubio, Abraham Isaac²; \\ Cruz-Palomares, Manueli3; González Vázquez, Annel ${ }^{4}$
}

\begin{abstract}
RESUMEN
Introducción: Con el aumento de la edad se presenta un descenso de las capacidades físicas, cognitivas y psicológicas, como respuesta a la necesidad de atención y cuidado han aparecido asilos o casa hogares para atender las necesidades de la población adulto mayor. Objetivo: identificar la influencia de la estancia en casa hogar o asilo en la calidad de vida del adulto mayor. Metodología: es una revisión bibliográfica, se guió por recomendaciones del protocolo PRISMA, mediante una búsqueda en distintas bases de datos electrónicas reconocidas, se identificaron 959 artículos primarios por región, la evaluación metodológica se utilizó el Checklist STROBE, después de utilizar los criterios se obtuvieron 18 artículos, se puede considerar que las casas hogar o asilos surgen para dar respuesta a los problemas y necesidades del adulto mayor, a pesar de esto dicha población presenta diversas complicaciones en sus condiciones de vida, además de desarrollar enfermedades y tener poco seguimiento por familiares; los adultos mayores califican su estancia como mala en general. Resultados y conclusiones: existe un deterioro de la calidad de vida del adulto mayor institucionalizado, que se implica en las funciones físicas, cognitivas y psico-emocionales, además de enfatizar la presencia de enfermedades crónico-degenerativas que representan un riesgo para la salud del adulto mayor y esperanza de vida. Se considera necesario promover políticas públicas que fomenten el envejecimiento saludable.
\end{abstract}

Palabras clave: Calidad de vida, Anciano, Hogares para ancianos (DeCS; BIREME).

1'Maestría en Ciencias de Enfermería, Profesor, Universidad Autónoma de Baja California, México. E-mail: atizoc@uabc.edu.mx

${ }^{2}$ Maestro en Enfermería, Profesor, Universidad Autónoma de Baja California, México

${ }^{3}$ Maestro en Ciencias de la Salud, Profesor, Universidad Autónoma de Baja California, México.

${ }^{4}$ Doctora en Ciencias de Enfermería, Universidad Autónoma de Nuevo León.

*Autor para correspondencia

\section{Cómo citar este artículo}

Tizoc-Márquez A, Esquivel-Rubio Al, Cruz-Palomares M, González-Vázquez A. Casa hogar-asilo y su influencia en la calidad de vida del adulto mayor: revisión bibliográfica. Sanus. 2018;3(6): 32-41. [Acceso_____]; Disponible en:

$$
\text { mes día año URL }
$$




\section{INTRODUCCIÓN}

Actualmente las personas con edades de 60 años han aumentado, alcanzando aproximadamente los 11.7 millones de personas a nivel mundial, esto se asocia a la disminución de fecundidad y la mortalidad a edades más avanzadas, situación que ha permitido un envejecimiento único en la historia. La Organización Mundial de la Salud (OMS), considera que esta situación ha sido posible por "éxito de las políticas de salud pública y el desarrollo socioeconómico"(1).

Entre 1930 y 2014, la esperanza de vida en México se ha duplicado, ganando 43 años en mujeres y 39 en hombre(2), lo cual ha implicado una mayor demanda de servicios de pensión, jubilación y salud, así como cambios en la organización familiar y cargas adicionales de trabajo para cuidadores. Para el año 2050 se prevé que las mujeres mayores de 60 años representen el 23.3\% y los hombres el 19.5\% ${ }^{(3)}$. Según el Consejo Nacional de población (CONAPO), en México habitaban 12.5 millones de adultos con edad superior a 65 años y de la cual se espera que para el año 2030 alcance un aproximado de 20.4 millones de personas ${ }^{(4)}$.

El aumento en la esperanza de vida, implica un reto para los sistemas de salud a nivel mundial, lo cual tiene efecto en las condiciones de vida del adulto mayor y por ende en la relación con la seguridad económica y la inclusión social de cada individuo ${ }^{(5)}$. El concepto de calidad de vida conlleva el análisis desde diversos puntos de vista, aunque de manera general, implica la autopercepción de la condición del individuo en aspectos sociales, culturales, espirituales y claro, sobre el estado de salud, ya sea física o psicológica, por lo que la dependencia en las acciones del día a día y la dinámica en que se determinan los nexos sociales, son en cierta medida, elementos esenciales para la obtención de este elemento(6).

Hoy en día, los hogares para ancianos han aparecido como respuesta a la necesidad de atención y cuidado del adulto mayor. Este segmento de población, ha llegado a ser considerado como una comunidad vulnerable, ya que en esta etapa de la vida, son propensos a sufrir un descenso de sus capacidades físicas, cognitivas y psicológicas ${ }^{(7)}$, hecho que ha llevado a establecer acciones normativas que aseguren condiciones óptimas de las instituciones que brinden servicios de asistencia a los adultos mayores, considerando que la población de adultos mayores está en incremento, es importante para el personal de salud conocer e identificar la forma en que la estancia de un adulto mayor en una casa hogar o asilo, influye en su calidad de vida.

Por ello la presente revisión bibliográfica tiene como objetivo describir la influencia de la estancia en casa hogarasilo en la calidad de vida del adulto mayor. A continuación se detalla la metodología para la búsqueda y análisis de artículos científicos.

\section{METODOLOGÍA}

Se trata de una revisión bibliográfica, el estudio se guió por las recomendaciones del protocolo PRISMA (por sus siglas en inglés Preferred Reporting Items for Systematic Reviews and Meta-Analyses) ${ }^{(8)}$, fueron incluidos estudios descriptivos correlacionales, en los idiomas inglés, español y portugués, en texto completo, que fueran realizados en adultos mayores que residen en casa hogar o asilo, la búsqueda no se limitó a estudios de años recientes.

Se realizó una búsqueda en las siguientes revistas electrónicas reconocidas del área de la salud, utilizando los Descriptores en Ciencias de la Salud (DeCS), combinados por medio de los operadores booleanos AND, OR y NOT, se identificaron 959 artículos primarios por región:

-Región Latinoamérica: Revista Latino-Americana de Enfermagem, Texto y Contexto Enfermagem, Revista da Escola de Enfermagem da U S P, Revista Enfermagem, Acta Paulista de Enfermagem, Online Brazilian Journal of Nursing, Ciencia y Enfermería (72).

-Región Norteamérica: The Gerontologist, Journals of Gerontology, Journal of Applied Gerontology, Journal of Housing for the Elderly, Clinical gerontologist, Activities, Adaptation and Aging, Physical and Occupational Therapy in Geriatrics (876).

-Región de Africa: Child Care in Practice, Sur African Journal of Clinical Nutrition, Africa Journal of Nursing and Midwifery (3).

-Región Europa: Journal of Food and Nutrition Research, Voprosy Pitania, Central European Journal of Nursing and Midwifery, European Journal of Mental Health Open Access, Advances in Gerontology, Revista Romana de Bioetica. Pediatria Wspolczesna (8).

Al realizar la revisión de cada artículo, se utilizaron distintos métodos de eliminación como: artículos duplicados, que no coincidían por título, no incluían variables relacionadas, no incluían objetivo claro, no estaban completos, los criterios de inclusión no coincidían con nuestro objetivo, entre otros, dejando como resultado la obtención de 18 artículo cientíicos para analizar a detalle.

En la evaluación de la calidad metodológica de los estudios seleccionados se utilizó el Checklist STROBE y un libro de $\operatorname{códigos}^{(9)}$. De esta forma la extracción de los datos fue obteniendo la información más importante que respondiera el objetivo de la revisión. Asimismo, se realizó la evaluación del sesgo, a través de una adecuación de la evaluación del sesgo expuesta en Cochrane Handbook for Systematic Reviews of Interventions ${ }^{(10)}$. 


\section{RESULTADOS}

Para fines de comprensión lectora, los hallazgos se presentan agrupados por temáticas afines:

\section{Salud mental}

Existen diversas situaciones asociadas a la salud mental de adultos mayores que residen en asilos, de los cuales destaca significativamente la depresión, los índices de esta afección mental en estudios realizados revelan lo siguiente: un indicativo de depresión del 42.8 \% y $35.7 \%$ en adultos mayores que residen dentro de un asilo(11), por otra parte se identifica un 33.3\% de depresión moderada(12), así mismo se presenta que en comparación el $42 \%$ de una población estudiada presentó niveles leves de depresión, $38 \%$ moderada y un 20\% niveles graves ${ }^{(13)}$, en función de lo reportado en 2015 los adultos mayores (39\%) presenta depresión moderada y el 23\% grave ${ }^{(14)}$, otras evidencias identifican que el $63.3 \%$ no demuestra sintomatologías de depresión, mientras que el 30\% tiene señales leves y $6.7 \%$ moderadas ${ }^{(15)}$. Por último, otro estudio relaciona estados de ánimo que presentan comúnmente en los adultos mayores dentro de estas instituciones como: tristeza: 51\%, melancolía: $32 \%$ y alegría: $15 \%$ (16)

\section{Autopercepción de la calidad de vida}

Dentro del análisis se puede describir que un 34\% de los adultos mayores residentes de casa hogar o asilo, califica su calidad de vida como muy mala, mientras que el 30\% lo califica normal, 33\% la considera buena y solo un 3\% la considera bastante buena ${ }^{(17)}$. En cuanto a la experiencia de estar dentro de una institución de cuidado, un 16.6\% expresa que se siente pésimo, el 50\% de la población se siente mal, el $22.2 \%$ se siente bien, y el $11.1 \%$ se siente muy bien ${ }^{(18)}$, mientras que otros datos señala que $82 \%$ de adultos mayores perciben baja calidad de vida con respecto a los años, es decir, a mayor edad menor calidad de vida ( $p=$ $0.211)$, además que a mayor escolaridad mayor la satisfacción de la vida $(p=0.989)^{(19)}$ vinculado a la temática otro estudio indica que un $56 \%$ presenta una calidad de vida baja ${ }^{(20)}$.

\section{Dependencia o capacidad funcional}

Algunos autores refieren que $64.28 \%$ de los ancianos son dependientes en acciones primordiales de la vida cotidiana ${ }^{(11)}$, sin embargo, otros perciben que el $77.5 \%$ presentó buena funcionalidad, mientras que el $13.7 \%$ disfuncionalidad moderada y el $8.8 \%$ severa ${ }^{(21)}$. En contraparte se muestra que el $19.8 \%$ presenta dependencia leve, y el $80.2 \%$ independencia ${ }^{(20)}$.

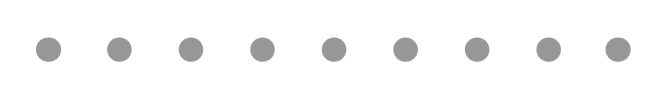

\section{Estado cognitivo}

Al respecto de éste ámbito, se refiere que el 58\% de adultos mayores institucionalizados presentaron deterioro cognitivo leve ${ }^{(12)}$, mientras que otras fuentes, proyectan que el $78.57 \%$ demuestran deterioro cognitivo moderado(11), en tanto que otra investigación señala un porcentaje de $43.12 \%$ con deterioro cognitivo y $21.6 \%$ con demencia en la población ${ }^{(14)}$. Un estudio realizado en Colombia, destaca que el estado cognitivo de una población con estas características, se considera bueno con un 98.5\%(22). Por otro lado, se identificó que el 39.8\% de un grupo de ancianos presentó deterioro cognitivo y al asociarlo con la variable sexo, mostró contraste entre hombres y mujeres, que obtuvieron en la escala MMSE, respectivamente, puntuaciones promedio de 14.8 y 12.8 ( $p=$ $0.0459)^{(23)}$.

\section{Patología asociada}

Se identificó que el porcentaje de enfermedades presentes en una población de adultos mayores, son: hipertensión arterial 50\%, diabetes mellitus 23.6\%, dislipidemias 22.3\% y osteoporosis $15 \%(p=0.010)^{(24)}$; otros autores muestran que el 30\% de la población padece HTA, un 13\% diabetes mellitus, mientras que el $5.2 \%$ no presentaban enfermedad ${ }^{(20)}$. En contra parte en otro grupo de estudio se describe que el $75 \%$ de su población presenta buena salud ${ }^{(22)}$.

\section{Situación familiar}

Para finalizar, en la esfera socioafectiva con relación a la convivencia familiar, diversos autores resaltan que el 58\% de los adultos mayores son abandonados en instituciones de cuidado por parte de su familia, y que el 51\% se manifiestan tristes, mientras que el $40 \%$ se adaptan a su nuevo hogar. Respecto a la frecuencia de las visitas por parte de familiares, el $50 \%$ recibe una visita al mes, mientras que el $28 \%$ no recibe visitas. También se identificó que la mitad de la población califica su relación familiar como mala, el 28\% pésima, el 17\% buena y solo el 5\% muy buena ${ }^{(18)}$. En índices estadísticos similares, se encuentra que el $47.31 \%$ recibe visita por parte de familiares dos veces al mes, el 29.03\% no tiene familia y el $17 \%$ no recibe visita alguna ${ }^{(25)}$.

\section{CONCLUSIONES}

La diversidad de resultados obtenidos apunta hacia la forma en como los adultos mayores que viven en una casa hogar-asilo presentan deterioro en la calidad de vida, situación que lleva a analizar y replantear las acciones que las instituciones tienen para el cuidado de esta población. Los autores concluyen que existe deterioro en la calidad de vida de la población mayor institucionalizada, siendo uno de los factores relevantes la ausencia del cuidado de la familia; además de ello sugieren que es de vital importancia fortalecer el vínculo familiar para evitar la depresión o el 
sentimiento de abandono, este último siendo un factor de riesgo para el deterioro progresivo de las funciones psicoemocionales.

Una gran cantidad de los adultos mayores que residen en una casa hogar-asilo, muestran dependencia en acciones cotidianas, además de manifestar enfermedades crónico-degenerativas que representan un riesgo para su salud y esperanza de vida.

Todo lo anterior, es relevante al momento de considerar que las instituciones que cuidarán de ellos, requieren de personal competente, de una infraestructura adecuada y de estrategias y planes de cuidado, que puedan satisfacer las necesidades y por ende, mejorar la calidad de vida. Se considera necesario el fortalecimiento de políticas que promuevan un envejecimiento saludable.

\section{REFERENCIAS BIBLIOGRÁFICAS}

1. Organización Mundial de la Salud. Informe Mundial sobre el envejecimiento y la salud [Internet]. Ginebra: Organización Mundial de la Salud; 2015 [Citado 25 Marzo 2018]. 282 p. Disponible en: http://apps.who.int/iris/bitstream/ handle/10665/186466/9789240694873_spa.pdf;jsessionid=0CA0E639487CFF10AE90D047D79B4438?sequence=1

2. Diario Oficial de la Federación. Plan Nacional de Desarrollo 2013-2018 [Internet]. 2013 [Citado 26 marzo 2018]. Disponible en: http://www.dof.gob.mx/nota_detalle_popup.php?codigo=5299465

3. Instituto Nacional de las Mujeres. Situación de las personas adultas mayores en México [Internet]. México: Instituto Nacional de las Mujeres; 2015 [Citado 27 Marzo 2017] 40 p. Disponible en: http://cedoc.inmujeres.gob.mx/documentos_ download/101243_1.pdf

4. Consejo Nacional de Población. La situación demográfica de México 2016 [Internet]. México: Consejo Nacional de Población; 2017 [Citado 28 marzo 2017]. 238 p. Disponible en: https://www.gob.mx/cms/uploads/attachment/file/253187/ SDM2016_web.pdf

5. Rubio Olivares DY, Rivera Martínez L, Borges Oquendo LC, González Crespo FV. Calidad de vida en el adulto mayor. Revista Científico- Metodológica [Internet]. 2015 [Citado 28 Marzo 2017];(61):1-7. Disponible en: http://www.redalyc.org/ html/3606/360643422019/

6. Martínez-Pérez TJ, González-Aragón CM, Castellón-León G, González-Aguiar B. El envejecimiento, la vejez y la calidad de vida: ¿éxito o dificultad? Revista Finlay [Internet]. 2018 [Citado 29 Marzo 2018];8(1):1-6. Disponible en: http://www.revfinlay.sld. cu/index.php/finlay/article/view/569

7. Montoya-Arce BJ, Román-Sánchez YG, Gaxiola-Robles-Linares SC, Montes de Oca-Vargas H. Envejecimiento y vulnerabilidad social en el Estado de México, 2010. Papeles de Población [Internet]. 2010 [Citado 25 Marzo 2017];22(90):43-77. Disponible en: https://rppoblacion.uaemex.mx/article/view/8256/6810

8. Liberati A, Altman DG, Tetzlaff J, Mulrow C, Gøtzsche PC, loannidis JP, et al. The PRISMA statement for reporting systematic reviews and meta-analyses of studies that evaluate health care interventions: explanation and elaboration. BMJ [Internet]. 2009 [Citado 29 Marzo 2017];339:1-27. Disponible en: https://www.bmj.com/content/bmj/339/bmj.b2700.full.pdf

9. Vandenbroucke JP, Von Elm E, Altman DG, Gøtzsche PC, Mulrow CD, Pocock SJ, et al. Strengthening the Reporting of Observational Studies in Epidemiology (STROBE): Explanation and Elaboration. PLoS Med [Internet]. 2007 [Citado 29 Marzo 2017];4(10):1628-54. Disponible en: http://journals.plos.org/plosmedicine/article/file?id=10.1371/journal. pmed.0040297\&type = printable

10. Higgins JP, Green S. Cochrane handbook for systematic reviews of interventions: [Internet]. Wiley; 2008 [Citado 29 Marzo 2017]. 672 p. Disponible en: https://www.wiley.com/engb/ Cochrane+Handbook+for+Systematic+Reviews+of+Interventions-p-9780470699515

11. Dávila JC, Buitrago JA, Ramos MA. Prevención y promoción de la salud física [Tesis de pregrado]. [Managua (NIC)]: Universidad Nacional Autónoma de Nicaragua; 2015. 66 p. Disponible en: http://repositorio.unan.edu.ni/2291/1/23869.pdf 12. López SE, Castillo FD, Carrazco SY, Medina AC, Ley-Wong LE. Comparación de calidad de vida en ancianos institucionalizados en asilos y no institucionalizados. Tepic. Nayarit. GEROINFO [Internet]. 2014 [Citado 23 Marzo 2017];9(2):1-13. Disponible en: http://www.medigraphic.com/pdfs/geroinfo/ger-2014/ger142c.pdf

13. Almeida MP. Análisis de los factores asociados a la depresión de los adultos mayores del hogar de ancianos "Santa Catalina Laboure", año 2012-2013 [Tesis de pregrado]. [Quito (EC)]: Pontificia Universidad Católica del Ecuador; 2013.102 p. Disponible en: http://repositorio.puce.edu.ec/bitstream/handle/22000/7224/8.14.001676.pdf?Sequence=4\&isallowed=y

14. Robalino MA. Repercusión del abandono en el estado mental y emocional del adulto mayor institucionalizado en los asilos de ancianos Sagrado corazón de Jesús de la ciudad de Ambato y el asilo de ancianos de Patate [Tesis de pregrado]. [Ambato (EC)]: Universidad Técnica de Ambato; 2015. 152p. Disponible en: http://repositorio.uta.edu.ec/jspui/ bitstream/123456789/9255/1/Robalino\%20Quintana\%2c\%20Miguel\%20Angel.pdf

15. Arias WL, Yepez L, Núñez A, Oblitas A, Pinedo S, Masías M, et al. Felicidad, depresión y creencia en la benevolencia humana en adultos mayores institucionalizados y no institucionalizados. Propósitos y Representaciones [Internet]. 2013 [Citado 1 Abril 2017];1(2):83-103. Disponible en: http://revistas.usil.edu.pe/index.php/pyr/article/view/32 
16. Quinteros MA. Factores de abandono y el buen vivir del adulto mayor del centro gerontológico "Señor de los Remedios" [Tesis de pregrado]. [Ambato (EC)]: Universidad Técnica de Ambato; 2015. 115 p. Disponible en: http://repositorio.uta.edu.ec/ jspui/bitstream/123456789/11568/1/FJCS-TS-177.pdf

17. Moya GC. Calidad de vida de los adultos mayores, del asilo de ancianos Señor de los Remedios, del Cantón Píllaro en el año 2016 [Tesis de pregrado]. [Ambato (EC)]: Universidad Regional Autónoma de los Andes; 2016. 104 p. Disponible en: http://dspace.uniandes.edu.ec/bitstream/123456789/5170/1/PIUAMED042-2016.pdf

18. Chamba GK. El Aislamiento Familiar y su Incidencia en la Determinación de la Calidad de Vida de los Adultos Mayores del Hogar de Ancianos "Dr. Hugo Agila Vargas", de la Ciudad de Catacocha Cantón Paltas desde el Enfoque del Trabajo Social [Tesis de pregrado]. [Loja (EC)]: Universidad Nacional de Loja; 2016. 160 p. Disponible en: http://dspace.unl.edu.ec/jspui/ bitstream/123456789/14835/1/Glenda\%20Karina\%20Chamba\%20Sánchez.pdf

19. Corugedo CM, García D, González VI, Crespo GA, González G, Calderín M. Calidad de vida en adultos mayores del hogar de ancianos del municipio Cruces. Revista Cubana de Medicina General Integral [Internet]. 2014 [Citado 22 Marzo 2017];30(2):208-16. Disponible en: http://www.medigraphic.com/pdfs/revcubmedgenint/cmi-2014/cmi142f.pdf

20. Loredo-Figueroa MT, Gallegos-Torres RM, Xeque-Molares AS, Palomé-Vega G, Juárez-Lira A. Nivel de dependencia, autocuidado y calidad de vida del adulto mayor. Enfermería universitaria [Internet]. 2016 [Citado 24 Marzo 2017];13(3):159-65. Disponible en: https://ac.els-cdn.com/S1665706316300197/1-s2.0-S1665706316300197-main.pdf?_tid=d48d131c-b24c-4350ac2c-0e1ef5a85f8c\&acdnat=1526608858_1ac362b2ad5e0efe1bced5ad02bb388e

21. Acuña I, Barrios Y, Martínez N, Taborda E, Vargas G. Percepción de la funcionalidad familiar en adultos mayores de los centros de vida de la ciudad de Cartagena [Tesis de pregrado]. [Cartagena (CO)]: Universidad de Cartagena; 2015. 51 p. Disponible en: http://repositorio.unicartagena.edu.co:8080/jspui/bitstream/11227/2642/1/PERCEPCI\%C3\%93N\%20 DE\%20LA\%2OFUNCIONALIDAD\%20FAMILIAR\%20EN\%20ADULTOS\%20MAYORES\%20DE\%20LOS\%20CENTROS\%20DE\%20 VIDA\%20DE\%20LA\%20CIUDAD\%20DE\%20CARTAGENA.pdf

22. Rocha M, Álvarez ME, Bayarre HD. Calidad de la atención al adulto mayor en el Hogar de ancianos "General Peraza". Revista Cubana de Medicina General Integral [Internet]. 2015 [Citado 27 Marzo 2017];31(2):143-58. Disponible en: http:// scielo.sld.cu/pdf/mgi/v31n2/mgi04215.pdf

23. Castro VC, Lange C, Baldissera VD, Silva ES, Carreira L. (2016). Cognitive assessment of elderly people in long-stay institutions: a cross-sectional study. Online Brazilian Journal of Nursing [Internet]. 2016 [Citado 20 Marzo 2017];15(3):372-81. Disponible en: http://www.objnursing.uff.br/index.php/nursing/article/view/5535/pdf_1

24. Cardona-Arias JA, Álvarez-Mendieta MI, Pastrana-Restrepo S. Calidad de vida relacionada con la salud en adultos mayores de hogares geriátricos, Medellín, Colombia, 2012. Revista Ciencias de la Salud [Internet]. 2014 [Citado 20 Marzo 2017];12(2):139-55. Disponible en: dx.doi.org/10.12804/revsalud12.2.2014.01

25. Bermeo DM, Guaicha BB. Nivel de satisfacción del adulto mayor respecto a los cuidados que reciben en el Asilo Cristo Rey de la ciudad de Cuenca, 2010 [Tesis de pregrado]. [Cuenca (EC)]: Universidad de Cuenca; 2010. 96 p. Disponible en: http:// dspace.ucuenca.edu.ec/handle/123456789/3570 